\title{
DEVELOPMENTS IN THE CLASSICAL NEVANLINNA THEORY OF MEROMORPHIC FUNCTIONS ${ }^{1,2}$
}

\author{
BY W. H. J. FUCHS
}

1. Nevanlinna's theory of meromorphic functions is about forty years old. The ideas of this theory reveal a "fine structure" of the distribution of values that was not visible to the older investigations of Picard, Borel and others. The aim of this paper is to report on some results concerning this "fine structure," especially on those based on the notion of Nevanlinna deficiency. It covers, therefore, only a very small part of the work on Nevanlinna Theory. In particular the many important generalizations of the classical Nevanlinna theory are not treated at all (Algebroid functions: H. Selberg [63], [64]; Meromorphic curves: L. Ahlfors [1], H. and J. Weyl [G]; Mappings of a Riemann surface in to another Riemann surface: S. S. Chern [7], L. Sario [60], [61] and (with K. Noshiro) [F]; Holomorphic mappings of complex analytic manifolds: R. Bott and S. S. Chern [6]).

2. By a meromorphic function $I$ shall mean a function meromorphic in $|z|<\infty$. The symbol $f(z)$ will always denote a meromorphic function. The core of Nevanlinna's Theory is expressed in the two "Fundamental Theorems." Some notation is needed for their statement. Let $n(r, f)$ denote the number of poles of $f(z)$ in $|z| \leqq r$, each pole counted with its proper multiplicity (simple pole once, double pole twice, $\cdots)$. Then the number of solutions of $f(z)=c$ in $|z| \leqq r$ is given by $n(r, 1 /(f-c))$. Let

$$
\begin{gathered}
N(r, \infty)=N(r, f)=\int_{0}^{r}(n(t, f)-n(0, f)) t^{-1} d t+n(0, f) \log r \\
m(r, \infty)=m(r, f)=(1 / 2 \pi) \int_{0}^{2 \pi} \log ^{+}\left|f\left(r e^{i \theta}\right)\right| d \theta \\
\cdot\left(\log ^{+}|n|=\max \{\log |n|, 0\}\right) . \\
N(r, c)=N(r, 1 /(f-c)), \quad m(r, c)=m(r, 1 /(f-c)) \quad(c \leqq \infty) .
\end{gathered}
$$

$N(r, c)$ is a smoothed counting function of the $c$-points of $f(z)$, $m(r, c)$ is a "proximity function" measuring how close $f(z)$ comes to $c$, on the average, on $|z|=r$. We can now state the

${ }^{1}$ An address given by Professor Fuchs before the meeting of the Society at New York University on February 26, 1966 by invitation of the Committee to Select Hour Speakers for Eastern Sectional Meetings; received by the editors December 12, 1966.

2 The author gratefully acknowledges support by the National Science Foundation. 
First Fundamental Theorem. For every complex number $c$ (1) $m(r, f)+N(r, f)=m(r, 1 /(f-c))+N(r, 1 /(f-c))+K(r, c)$, where

$$
|K(r, c)|<2+\log ^{+}|c| .
$$

The interest lies here in the behavior of both sides of (1) as $r \rightarrow \infty$. It turns out that both sides tend to infinity, so that $K(c, r)$ is a negligible error-term.

Proofs of the Fundamental Theorems can be found in Hayman's monograph [A].

Definition 1. The function

$$
T(r, f)=m(r, f)+N(r, f)
$$

is the (Nevanlinna) characteristic function of $f(z)$. In the Nevanlinna theory the characteristic function takes the role played by

$$
\log M(r, f)=\underset{|z| \leqq r}{\log \sup }|f(z)|
$$

in the older theory of entire functions.

$T(r, f)$ is an increasing, convex function of $\log r$ tending to $\infty$ for every nonconstant $f$. Unless $f(z)$ is a rational function

$$
T(r, f) / \log r \rightarrow \infty \quad(r \rightarrow \infty) .
$$

and, for constant $\alpha, \beta, \gamma, \delta$,

$$
T(r,(\alpha f+\beta) /(\gamma f+\delta))=T(r, f)+O(1) \quad(\alpha \delta-\beta \gamma \neq 0) .
$$

Definition 2. The order $\rho$ of $f(z)$ is given by

$$
\rho=\limsup _{r \rightarrow \infty}(\log T(r, f)) / \log r .
$$

The lower order $\lambda$ of $f(z)$ is given by

$$
\lambda=\liminf _{r \rightarrow \infty}(\log T(r, f)) / \log r .
$$

For an entire function $g(z)$

$$
T(r, g) \leqq \log M(r, g) \leqq(R+r) /(R-r) T(R, g) \quad(R>r) .
$$

Also, if the lower order $\lambda$ of $g$ satisfies $0 \leqq \lambda \leqq \frac{1}{2}$, then (Ostrowski [52]; with $\rho$ in place of $\lambda$ Valiron [67]);

$$
\underset{r \rightarrow \infty}{\liminf \log M(r, g) / T(r, g) \leqq \pi \lambda \operatorname{cosec} \pi \lambda .}
$$


For $\lambda>\frac{1}{2}$ the best-possible value on the right-hand side of (3) is probably $\pi \lambda$, but this has not yet been proved. However, V. P. Petrenko [57] showed that for every real $\phi$

$$
\lim \inf \log \left|g\left(r e^{i \phi}\right)\right| / T(r, g) \leqq \pi \lambda \quad(\lambda>1 / 2),
$$

following an earlier paper by A. A. Goldberg [32] where this is proved with $\rho$ in place of $\lambda$. Examples of R. E. A. C. Paley [54] show that for every order $\rho$ there are entire functions with

$$
\limsup _{r \rightarrow \infty} \log M(r, g) / T(r, g)=\infty .
$$

3. Second Fundamental Theorem. Let $c_{1}, c_{2}, \cdots, c_{q}$ be $q(\geqq 2)$ complex numbers. Then

$$
m(r, f)+\sum_{\nu=1}^{q} m\left(r, 1 /\left(f-c_{\nu}\right)\right) \leqq 2 T(r, f)-N_{1}(r)+S(r),
$$

where

$$
N_{1}(r)=N\left(r, 1 / f^{\prime}(z)\right)+2 N(r, f(z))-N\left(r, f^{\prime}(z)\right)
$$

is the smoothed counting function of multiple points (a point of multiplicity $p$ is counted $p-1$ times) and

$$
S(r)=O(\log r+\log T(r))
$$

for all $r$ outside a set of finite measure.

If $f(z)$ is of finite order, then

$$
S(r)=O(\log r)
$$

is true for all large $r$.

The term $N_{1}(r)$ in (4) gives rise to many interesting theorems about multiple values; in this paper it will be sufficient to estimate it by the obvious inequality

$$
N_{1}(r) \geqq 0 \quad(r>1) .
$$

Dividing (4) by $T(r, f)$ and letting $r \rightarrow \infty$ through a suitable sequence of values yields the important

Corollary. If $c_{1}, c_{2}, \cdots, c_{k}$ are $k(\geqq 3)$ distinct values ( $\infty$ is not excluded), then

$$
\sum_{\nu=1}^{k} \liminf _{r \rightarrow \infty} \frac{m\left(r, c_{\nu}\right)}{T(r, f)} \leqq \liminf _{r \rightarrow \infty} \sum_{\nu=1}^{k} \frac{m\left(r, c_{\nu}\right)}{T(r, f)} \leqq 2 .
$$


Definition 3. The number $\delta(c, f)=\lim \inf _{r \rightarrow \infty} m(r, c) / T(r, f)$ is the (Nevanlinna) deficiency of $c$ with respect to $f(z)$.

By the First Fundamental Theorem

$$
\begin{aligned}
\delta(c, f) & =\liminf _{r \rightarrow \infty} m(r, c) / T(r, f) \\
& =1-\limsup _{r \rightarrow \infty} N(r, c) / T(r, f) .
\end{aligned}
$$

It follows at once that

$$
0 \leqq \delta(c, f) \leqq 1 .
$$

Definition 4. The value $c$ is deficient with respect to $f(z)$, if

$$
\delta(c, f)>0 .
$$

The inequality (5) yields easily

THEOREM 1. A meromorphic function $f(z)$ has, at most, a finite or denumerable set of deficient values and

$$
\sum_{c} \delta(c, f) \leqq 2,
$$

where the summation is over all deficient values.

If a value $c$ is not assumed by $f(z)$, then, by $(6), \delta(c, f)=1$. Theorem 1 contains therefore the sharpened form of

Picard's Theorem. If $f(z) \neq a$ and $f(z) \neq b$, then $\delta(c, f)=0$ for every $c$ different from $a$ and $b$.

4. The study of many types of special functions led R. Nevanlinna to the following three

Conjectures. (a) Deficient values are asymptotic values,

(b) $m\left(r, f^{\prime}\right) \sim m(r, f)$,

(c) the number of deficient values of a meromorphic function is finite.

Mme. L. Schwartz and O. Teichmüller [62], [66] independently disproved conjecture (a) by giving examples of meromorphic functions with a deficient value which is not an asymptotic value. W. Hayman and A. Goldberg gave examples of entire functions with this property [39], [27]. Goldberg constructed such entire functions of order $1+\epsilon$ for every positive $\epsilon$.

In all these examples the deficiencies are rather small. At the International Congress of Mathematicians, Moscow, 1966, A. A. 
Goldberg announced an example of a meromorphic function with $\delta(0, f)=1$, for which 0 is not an asymptotic value.

Conjecture (b) has been thoroughly investigated and disproved in two fairly recent papers by W. K. Hayman [40], [41].

The first counterexample to conjecture (c) was given by A. A. Goldberg who gave an example of a meromorphic function with infinitely many deficient values [24]. His example was moreover of finite order. Later on he refined his construction to show that this order could be chosen arbitrarily small.

Quite recently N. U. Arakelyan [4] proved that there are entire functions of every order $\rho>\frac{1}{2}$ with infinitely many deficient values. The lower bound $\frac{1}{2}$ is best-possible: By a well-known theorem of Wiman an entire function $g(z)$ of order $<\frac{1}{2}$ has the property that $\left|g\left(r_{n} e^{i \theta}\right)\right| \rightarrow \infty$ uniformly in $\theta$ as $r_{n} \rightarrow \infty$ through a suitable sequence of values. This implies $m\left(r_{n}, c\right)=0$ for every complex $c$ and $n>n_{0}(c)$, so that $\delta(c, f)=0$.

5. It is natural to ask whether the deficiencies $\delta(c, f)$ are subject to any restrictions beyond those expressed in Theorem 1. A complete, negative answer is known in the case of entire functions:

Theorem 2 (Fuchs-Hayman [22]). Given a finite or denumerable set of complex numbers $c_{1}, c_{2}, \cdots$, and a set of real numbers $\delta_{j}=\delta\left(c_{j}\right)$ subject to $0<\delta_{j} \leqq 1, \sum \delta_{j} \leqq 1$, it is possible to find an entire function $f(z)$ such that

$$
\delta\left(c_{j}, f\right)=\delta_{j},
$$

and $\delta(b, f)=0$ for every $b$ which does not belong to the set $\left\{c_{j}\right\}$.

There is in all probability an analogous theorem for meromorphic functions (now $\sum \delta_{j} \leqq 2$ ), but this has not been proved, to my knowledge. For the case of a finite number of deficient values with sum $<2$ the analogue is true (Goldberg [23]).

The situation becomes much more complicated, if attention is restricted to functions of finite order or of finite lower order. In this case the $\delta$ are subject to further restrictions which are only incompletely known at present.

Theorem 3 (HAyman [A, TheOREM 4.2]). If $f(z)$ is a nonconstant meromorphic function of finite lower order $\lambda$ and if $\epsilon>0$, then there is a constant $K(\lambda, \epsilon)$ such that

$$
\sum(\delta(c, f))^{1 / 3+\epsilon}<K(\lambda, \epsilon) .
$$

In this theorem the $\frac{1}{3}$ in the exponent cannot be replaced by a 
smaller number, since there are meromorphic functions with $\sum(\delta(a, f))^{1 / 3-\epsilon}=\infty(\epsilon>0)$. However, Arakelyan conjectures that in the case of entire functions stronger inequalities are true.

6. An interesting open problem is the investigation of the least upper bound of

$$
\Delta(f)=\sum_{c} \delta(c, f)
$$

as $f$ runs through all meromorphic functions of a given order $\rho$ or lower order $\lambda$.

A way of attacking this problem is provided by the following lemma which is a simple byproduct of Nevanlinna's proof of the Second Fundamental Theorem.

Lemma 1 (Wittich [E]). For entire functions of finite order

$$
\Delta(f) \leqq \delta\left(0, f^{\prime}\right)+\delta\left(\infty, f^{\prime}\right)=\delta\left(0, f^{\prime}\right)+1
$$

for meromorphic functions of finite order

$$
\Delta(f) \leqq 2\left\{\delta\left(0, f^{\prime}\right)+\delta\left(\infty, f^{\prime}\right)\right\} .
$$

Upper bounds for $\Delta$ can therefore be derived from lower bounds of (7) $2-\delta(\infty)-\delta(0) \geqq \limsup _{r \rightarrow \infty}(N(r, f)+N(r, 1 / f)) / T(r, f)=k(f)$.

Nevanlinna made the

CONJECTURe 1. For every meromorphic function $f(z)$ of order $\rho<\infty$

$$
\begin{aligned}
k(f) & \geqq|\sin \pi \rho| /([\rho]+|\sin \pi \rho|) \quad[\rho] \leqq \rho \leqq[\rho]+1 / 2, \\
& \geqq|\sin \pi \rho| /([\rho]+1) \quad[\rho]+1 / 2 \leqq \rho \leqq[\rho]+1 .
\end{aligned}
$$

A. Edrei [10] and I. V. Ostrovskii [53] proved independently Theorem 4. Let

$$
\begin{aligned}
\nu(x) & =1 \quad(0 \leqq x \leqq 1 / 2), \\
& =\sin \pi x \quad(1 / 2 \leqq x \leqq 1), \\
& =|\sin \pi x| /(A x+1 / 2|\sin \pi x|) \quad(1<x),
\end{aligned}
$$

where $A<12$ is an absolute constant.

If $f(z)$ is a meromorphic function of order $\rho$ and lower order $\lambda$, then

$$
k(f) \geqq \sup _{\lambda \leqq x \leqq \rho} \nu(x),
$$

where $k$ is defined by (7). 
In some special cases the answer to the problem raised at the beginning of this section is known. Quite recently A. Edrei proved the remarkable

THEOREM 5 [11]. If $f(z)$ is a meromorphic function of lower order $\lambda<\frac{1}{2}$, then

$$
\Delta(f) \leqq 1 .
$$

This upper bound is attained, if and only if $f(z)$ has one deficient value $c$ with $\delta(c, f)=1$. If $f(z)$ has at least two deficient values, then

$$
\Delta(f)<1-\cos \pi \lambda .
$$

Edrei's paper also contains

CONJECTURE 2. If $\frac{1}{2}<\lambda \leqq 1$,

$$
\Delta(f) \leqq 2-\sin \pi \lambda .
$$

A. Pfluger has investigated under which circumstances the sum of the deficiencies of an entire function can reach the maximum value 2 . $\mathrm{He}$ obtained the beautiful

THEOREM 6 [58]. If $f(z)$ is an entire function of finite order $\rho$ with $\sum_{c} \delta(c, f)=2$, then $\rho$ is a positive integer and, for every deficient value $c, \delta(c, f)$ is an integral multiple of $1 / \rho$. In particular there cannot be more than $\rho$ finite deficient values.

Additional information is given by

TheOREM 7 (EDREI-FuCHS [14]). Given $\epsilon, 0<\epsilon<\frac{1}{2}$, and $\lambda>0$, there is a $\delta=\epsilon \delta_{1}(\lambda)$ such that every entire function $f(z)$ of lower order $\lambda$ with $\sum_{c} \delta(c, f)>2-\delta$ has the following properties:

(a) There is a positive integer $p$ such that $p$ differs from the order $\rho$ of $f(z)$ and from the lower order $\lambda$ by less than $\epsilon$.

(b) There are $s \leqq p$ finite deficient values $c_{1} \cdots c_{s}$ such that each $\delta\left(c_{j}, f\right)$ differs by less than $A_{1} \in$ from an integral multiple of $1 / p$ and $\sum_{k=1}^{s} \delta\left(c_{k}, f\right)>1-A_{2} \epsilon\left(A_{1}\right.$ and $A_{2}$ absolute constants $)$.

(c) Each $c_{k}$ is an asymptotic value of $f(z)$.

The analogue of Theorem 6 for meromorphic functions is likely to be:

CONJECTURE 3. If $f(z)$ is a meromorphic function of finite order with $\Delta(f)=2$, then the order $\rho$ of $f(z)$ is of the form $\rho=n / 2$ $(n=2,3,4, \cdots)$; all deficiencies are multiples of $1 / \rho$; in particular $f(z)$ has only finitely many deficient values.

Recently Edrei proved 
THEOREM 8 [12]. If $f(z)$ is a meromorphic function of lower order $\lambda \leqq 1$, then $\Delta(f)=2$ implies that $f(z)$ has either two or three deficient values. Also $\lambda \geqq 5 / 6$.

It is very likely that the case of three deficient values cannot occur. If this is so, then $\lambda=1$.

7. Several results are known for functions of lower order $\lambda<1$.

Their proofs are based on elaborations of the following simple remark: Let

$$
f(z)=\Pi\left(1-z / a_{k}\right) / \Pi\left(1-z / b_{k}\right)
$$

be an entire function of order $\rho<1$. If for a given $r>0$ $E=\left\{\theta|| f\left(r e^{i \theta}\right) \mid>1\right\}$, then

$$
m(r, f)=\frac{1}{2 \pi} \sum \int_{E} \log \left|1-\frac{r e^{i \theta}}{a_{k}}\right| d \theta-\frac{1}{2 \pi} \sum \int_{E} \log \left|1-\frac{r e^{i \theta}}{b_{k}}\right| d \theta .
$$

If the measure of $E$ is $2 \beta(0 \leqq \beta \leqq \pi)$, then

$$
\frac{1}{2 \pi} \int_{E} \log \left|1-\frac{r e^{i \theta}}{a_{k}}\right| d \theta \leqq \frac{1}{2 \pi} \int_{-\beta}^{\beta} \log \left|1+\frac{r e^{i \theta}}{\left|a_{k}\right|}\right| d \theta
$$

since $\log \left|1-\rho e^{i \theta}\right|(0 \leqq|\theta|<\pi, \rho>0)$ is an increasing function of $|\theta|$ in $0 \leqq|\theta| \leqq \pi$. Similarly

$$
\frac{1}{2 \pi} \int_{E} \log \left|1-\frac{r e^{i \theta}}{b_{k}}\right| d \theta \geqq \frac{1}{2 \pi} \int_{-\beta}^{+\beta} \log \left|1-\frac{r e^{i \theta}}{\left|b_{k}\right|}\right| d \theta
$$

and so

$$
m(r, f) \leqq \frac{1}{2 \pi} \int_{-\beta}^{\beta} \log \left|f_{1}\left(r e^{i \theta}\right)\right| d \theta
$$

where

$$
f_{1}(z)=\Pi\left(1+z /\left|a_{k}\right|\right) / \Pi\left(1-z /\left|b_{k}\right|\right) .
$$

Since $\left|f_{1}\left(r e^{i \theta}\right)\right|$ is a decreasing function of $|\theta|$ in $0 \leqq|\theta| \leqq \pi$, there is a $\gamma, 0 \leqq \gamma \leqq \pi$, such that

$$
\left|f_{1}\left(r e^{i \theta}\right)\right|>1 \quad(|\theta|<\gamma), \quad\left|f_{1}\left(r e^{i \theta}\right)\right|<1 \quad(\gamma<|\theta|<\pi) .
$$

Obviously

$$
m\left(r, f_{1}\right)=\frac{1}{2 \pi} \int_{-\gamma}^{\gamma} \log \left|f_{1}\left(r e^{i \theta}\right)\right| d \theta \geqq \frac{1}{2 \pi} \int_{-\beta}^{\beta} \log \left|f_{1}\left(r e^{i \theta}\right)\right| d \theta \geqq m(r, f) .
$$


Since $N\left(r, f_{1}\right)=N(r, f)$,

$$
\begin{aligned}
\delta\left(\infty, f_{1}\right) & =\lim \inf m\left(r, f_{1}\right) /\left(m\left(r, f_{1}\right)+N(r, f)\right) \\
& \geqq \lim \inf m(r, f) /(m(r, f)+N(r, f))=\delta(\infty, f) .
\end{aligned}
$$

By the same method we have also $\delta\left(0, f_{1}\right) \geqq \delta(0, f)$ (A. Goldberg [26], with a different proof). This reduces the estimation of $\delta(0, f)$ and $\delta(\infty, f)$ to the case of functions with real, positive zeros and real, negative poles. In this case the relation between the size of the deficiencies and the angle $\beta$ can be worked out in fair detail. This leads to

LEMMA 2. If $K>0$ and if $f(z)$ is a meromorphic function of lower order $\lambda<1$ then the set of numbers

$$
\beta(r)=(1 / 2) m\left\{\theta|| f\left(r e^{i \theta}\right) \mid>K\right\}
$$

has a limit point $\beta$ such that

$$
\sin \pi \lambda \leqq(1-\delta(0, f)) \sin \beta \lambda+(1-\delta(\infty, f)) \sin (\pi-\beta) \lambda .
$$

An immediate consequence is (Edrei [10], Ostrovskii [52]).

THEOREM 9. If $f(z)$ is a meromorphic function of lower order $\lambda<1$ and if $a$ and $b$ are two points of the extended complex plane,

$$
u=1-\delta(a, f), \quad v=1-\delta(b, f),
$$

then

$$
0 \leqq u \leqq 1, \quad 0 \leqq v \leqq 1
$$

and

$$
u^{2}+v^{2}-2 u v \cos \pi \lambda \geqq \sin ^{2} \pi \lambda .
$$

If $u \leqq \cos \pi \lambda$, then $v=1$.

For any pair of numbers $u_{0}, v_{0}$ obeying the restrictions just stated there is a meromorphic function of order $\lambda$ for which $u=u_{0}, v=v_{0}$.

More precise information about functions with a deficiency $\delta(a, f)$ $\geqq 1-\cos \pi \lambda$ is contained in

Theorem 10 (OstrovskiI [52]; EdReI [10]). Let $f(z)$ be a meromorphic function of lower order $\lambda<\frac{1}{2}$. Let

$$
\mu(r, f)=\inf _{|z|=r}|f(z)| .
$$

Then

$$
\limsup _{r \rightarrow \infty} \frac{\log ^{+} \mu(r, f)}{T(r, f)} \geqq \frac{\pi \lambda}{\sin \pi \lambda}\{\cos \pi \lambda-1+\delta(\infty, f)\} .
$$


Corollary. If $\delta(\infty, f)>1-\cos \pi \lambda$, then $\left|f\left(r e^{i \theta}\right)\right| \rightarrow \infty$, uniformly in $\theta$, as $r_{n} \rightarrow \infty$ through a suitable sequence. In particular $f(z)$ has no finite deficient value.

Theorem 10 is, of course, closely connected to Wiman's Theorem on entire functions of order less than $\frac{1}{2}$. This theorem, in the improved form given to it by Kjellberg [43], states

THEOREM 11. Let $f(z)$ be an entire function of lower order $\lambda<1$. Then $\lim \sup (\log \mu(r, f) / \log M(r, f)) \geqq \cos \pi \lambda$.

Recently much work has been done on the closer investigation of entire functions of lower order $\lambda<1$ (M. Anderson [2], [3], M. Essén [19]). Most of this work arose from

Theorem 12 (KJELLberg [44]). Let $0<\sigma<1$. Let $f(z)$ be an entire function such that

$$
\log \mu(r, f)-\cos \pi \sigma \log M(r, f) \leqq 0 \quad\left(r>r_{0}\right) .
$$

Then $L=\lim _{r \rightarrow \infty} r^{-\sigma} \log M(r)$ exists and $0<L \leqq \infty$.

8. An extension of Theorem 10 in one direction is

THEOREM 13 (EDREI [11]). Let $f(z)$ be a meromorphic function of lower order $\lambda$ and let $\delta(c, f)>0$. If the nonnegative integer $q$ is chosen so that

$$
\cos (\pi \lambda / q) \geqq 1-\delta(\tau, f), \quad q \geqq 2 \lambda,
$$

then there is a sequence $\left\{r_{n}\right\}, r_{n} \rightarrow \infty$, such that for every $\eta>0$

$$
\lim \inf m\left\{\theta|| f\left(r_{n} e^{i \theta}\right)-c \mid<\eta\right\} \geqq 2 \pi / q .
$$

The sequence $r_{n}$ depends only on $T(r, f)$, not on the choice of $c$ or $\eta$.

Theorem 13 is the basis of the proofs of Theorems 5 and 8 . It also lends support to the following conjecture which was enunciated in a weaker form by Teichmüller [66].

CONJECTURE 4. If $f(z)$ is a meromorphic function of lower order $\lambda$, then there is a sequence $\left\{r_{n}\right\}, r_{n} \rightarrow \infty$, such that for every deficient value $c$ of $f$ and every $\eta>0$

$$
\liminf _{n \rightarrow \infty} m\left\{\theta|| f\left(r_{n} e^{i \theta}\right)-c \mid<\eta\right\} \geqq \inf \{2 \pi,(2 / \lambda) \arccos (1-\delta(c, f))\} .
$$

The conjecture is true, by Theorem 13 , if $\delta(c, f)=1-\cos (\pi \lambda / q), q$ a positive integer $\geqq 2 \lambda$.

The truth of Conjecture 4 would imply the truth of Conjecture 2 .

Conjecture 4 was verified by Teichmüller for a very special class 
of functions. It receives further support from

TheOREM 14 (ARIMA [5]). Let $f(z)$ be an entire function of order $\rho$. Let $\alpha(r)$ be the angle subtended at the origin by the largest arc of $|z|=r$ on which $|f(z)|>1$. Then

$$
\limsup _{r \rightarrow \infty} \alpha(r) \geqq \pi / \rho .
$$

Notice also that Theorem 9 would be an immediate consequence of Conjecture 4. Its inequalities express the obvious fact that the sets $\left\{\theta|| f\left(r e^{i \theta}\right)-a \mid<\eta\right\}$ and $\left\{\theta|| f\left(r e^{i \theta}\right)-b \mid<\eta\right\}$ are disjoint and of total measure $\leqq 2 \pi$, if $\eta$ is sufficiently small.

9. Since the definition of the $N$-function and therefore also of $\delta(c, f)$ depends only on the absolute values of the $c$-points of $f(z)$, it is surprising that knowledge of the angular distribution of $c$-points gives information about deficiencies.

Theorem 15 (EdRer [8], EdRei ANd Fuchs [16], [17]). If all the zeros and poles of the meromorphic function $f(z)$ lie on $s \geqq 1$ radial lines, then $f(z)$ has at most $s$ deficient values other than 0 and $\infty$. If the maximum number $s$ is attained, each deficient value must be asymptotic.

The total number of deficient values is at most $s+1$, this maximum can only be attained if $\delta(0, f)>0$ and $\delta(\infty, f)>0$.

If $\delta(c, f)>0, c \neq 0, \infty$, and if $\alpha$ is the minimum angle between two radial lines carrying zeros or poles, then the order $\rho$ of $f(z)$ is less than $\pi / \alpha$.

If $f(z)$ is of order $\rho$, then the number of deficient values other than 0 and $\infty$ is less than $\min (s, 2 \rho)$.

Many generalizations of this theorem are known. The zeros and poles need not be exactly on the lines, it is enough that they are close to the lines (Ostrovskii [51]). The radial lines may be replaced by curves of certain types (Edrei and Fuchs [17]), the angular condition need only be satisfied in some, rather narrow, annuli (Edrei and Fuchs [16]).

Theorem 16 (EDrei, Fuchs ANd Hellerstein [18]). Let all zeros $a_{k}$ of the entire function $f(z)$ lie on a finite number of radial lines. There is a constant $k$ depending only on the configuration of these lines so that

$$
\sum\left|a_{n}\right|^{-k}=\infty, \quad \sum\left|a_{n}\right|^{-\xi}<\infty \quad \text { for some } \xi
$$

implies $\delta(0, f)>A$, where $A$ is an absolute constant.

Generalizations to meromorphic functions are given in [42]. 
Theorem 17 (Ostrovskir [50]). If $f(z)$ is of order $\rho$ and all poles and zeros of $f(z)$ lie in a sector

$$
\alpha \leqq \arg z \leqq \alpha+\gamma
$$

where

$$
\pi /(2 \pi-\gamma)<\rho<\pi / \gamma
$$

then

$$
\delta(c, f)<1-\cos (\gamma \rho / 2) \quad(c \neq 0, \infty) .
$$

10. There are relations between the deficiencies and the structure of the power series of an entire function.

Theorem 18. If

$$
f(z)=\sum_{1}^{\infty} c_{k} z^{n_{k}}
$$

is an entire function of finite order and if

$$
n_{k} / k \rightarrow \infty \quad(k \rightarrow \infty),
$$

then $f(z)$ has no finite deficient value.

This is an immediate consequence of the fact that $f\left(r e^{i \theta}\right) \rightarrow \infty$, uniformly in $\theta$, as $r \rightarrow \infty$ through a suitable sequence of values [21].

In the case of functions of infinite order a similar theorem may hold with (9) replaced by the condition $\sum 1 / n_{k}<\infty$. At present it is only known that under this condition $f(z)$ assumes every finite value infinitely often. Kövari [45] has proved (implicitly)

THEOREM 19. If $f(z)$ is an entire function with the power series (8) and if

$$
\lambda_{n}>n(\log n)^{2+n}
$$

then

$$
\delta(c, f)=0 \quad(c \neq \infty) .
$$

M. Anderson (unpublished) proved

THEOREM 20. If $f(z)$, given by (8), is an entire function of lower order $\lambda<\infty$ and if every circle $|z|=r, r>r_{0}$, contains a point at which $|f(z)|<K$, for some positive $K$, then

$$
\lim \inf \left(n_{k+1}-n_{k}\right) \leqq C(\lambda) \quad(k \rightarrow \infty),
$$

where the function $C(\lambda)$ satisfies 


$$
C(\lambda)=O\left(\lambda^{2} \log ^{+} \lambda+1\right) .
$$

It follows that $f(z)$ can not have a finite deficient value, if $\lim \inf \left(n_{k+1}-n_{k}\right)>C(\lambda)$.

THEOREM 21. If the set of exponentials $\left\{e^{i n_{k} t}\right\}_{k=1}^{\infty}$ is not complete in $L^{2}(-D, D)$, then all deficiencies of an entire function of lower order $\lambda$ with the expansion (8) satisfy

$$
\delta(c, f)<A \lambda D \quad(c \neq \infty),
$$

where $A$ is an absolute constant.

The proof of this theorem is based on Petrenko's improvement of an earlier result $[20]$ :

THEOREM 22 [55]. If $f(z)$ is a meromorphic function of lower order $\lambda>0$, then for some arbitrarily large $r$

$$
r \int_{0}^{2 \pi}\left|f^{\prime}\left(r e^{i \theta}\right) / f\left(r e^{i \theta}\right)\right| d \theta<B \lambda T(r, f),
$$

where $B$ is an absolute constant.

A generalization of an earlier result of A. Pfluger and G. Pólya [59] on entire functions with a Borel-deficient value is

TheOREM 23 (EDReI AND Fuchs [14]). Let $f(z)$ be an entire function of lower order $\lambda$ with the power series (8). There is an absolute constant A such that

$$
\sum_{c} \delta(c, f)>2-A \epsilon /(\lambda+1)(1+\log (\lambda+1))
$$

has the following consequences: The order $\rho$ and the lower order $\lambda$ are close to an integer $p$. If

$$
\begin{aligned}
& D=\liminf _{N \rightarrow \infty}(1 / N) \sum_{n_{k} \leq N}\left(1 / n_{k}\right) \\
& \bar{D}=\limsup _{N \rightarrow \infty}(1 / N) \sum_{n_{k} \leq N}\left(1 / n_{k}\right),
\end{aligned}
$$

then there is an integer $s, 1 \leqq s \leqq p$, such that

$$
(1-\epsilon) s / p \leqq D \leqq \bar{D} \leqq s / p .
$$

\section{REFERENCES}

\section{TEXTBOOKS OF NEVANLINNA THEORY}

A. W. K. Hayman, Meromorphic functions, Oxford Univ. Press, New York, 1964 
B. K. L. Hiong, Sur les fonctions méromorphes et les fonctions algêbroides, Mémor. Sci. Math. no. 139, Gauthier-Villars, Paris, 1957.

C. R. Nevanlinna, Le theorème de Picard-Borel et la theorie des fonctions meromorphes, Gauthier-Villars, Paris, 1929.

D. - Eindeutige analytische Funktionen, 2nd ed., Springer, Berlin, 1953.

E. H. Wittich, Neuere Untersuchungen über eindeutige analytische Funktionen, Springer, Berlin, 1955.

F. L. Sario and K. Noshiro, Value distribution theory, Van Nostrand, Princeton, N. J., 1966.

G. H. Weyl and J. Weyl, Meromorphic functions and analytic curves, Princeton Univ. Press, Princeton, N. J., 1943.

\section{PAPERS}

1. L. V. Ahlfors, The theory of meromorphic curves, Acta Soc. Sci. Fenn. A(2) 3, No. 4 (1941), 31 pp.

2. J. M. Anderson, Asymptotic properties of integral functions of genus zero, Quart. J. Math. Oxford Ser. (2) 16 (1965), 151-164.

3. - Growth properties of integral and sub-harmonic functions, J. Analyse Math. 13 (1964), 355-389.

4. N. U. Arakelyan, Entire functions of finite order with infinitely many deficient values, International Congress of Mathematicians, Moscow, 1966.

5. K. Arima, On maximum modulus of integral functions, J. Math. Soc. Japan 4 (1952), 62-66.

6. R. Bott and S. S. Chern, Hermitian vector bundles and the equidistribution of the zeros of their holomorphic sections, Acta Math. 114 (1965), 71-112.

7. S. S. Chern, Complex analytic mappings of Riemann surfaces. I, Amer. J. Math. 82 (1960), 323-337.

8. A. Edrei, Meromorphic functions with three radially distributed values, Trans. Amer. Math. Soc. 78 (1955), 276-293.

9. - Meromorphic functions with values that are both deficient and asymptotic, Studies in Mathematical Analysis and Related Topics, Stanford Univ. Press, Stanford, Calif., 1962.

10. - The deficiencies of functions of finite lower order, Duke Math. J. 31 (1964), 1-22.

11. - Sums of deficiencies of meromorphic functions, J. Analyse Math. 14 (1965), 79-107.

12. - Sums of deficiencies of meromorphic functions. II, J. Analyse Math. (to appear).

13. A. Edrei and W. H. J. Fuchs, On the growth of meromorphic functions with several deficient values, Trans. Amer. Math. Soc. 93 (1959), 292-328.

14. - Valeurs deficientes et valeurs asymptotiques des fonctions meromorphes, Comment. Math. Helv. 33 (1959), 258-295.

15. - On the deficiencies of meromorphic functions of order less than one, Duke Math. J. 27 (1960), 233-250.

16. - Bounds for the number of deficient values of certain classes of meromorphic functions, Proc. London Math. Soc. 12 (1962), 317-344.

17. - On meromorphic functions with regions free of poles and zeros, Acta Math. 108 (1962), 113-145.

18. A. Edrei, W. H. J. Fuchs, and S. Hellerstein, Radial distribution and the deficiencies of the values of a meromorphic function, Pacific J. Math. 11 (1961), 135-151. 
19. M. Essen, $A$ theorem on the maximum modulus of entire functions, Math. Scand. 17 (1965), 161-168.

20. W. H. J. Fuchs, $A$ theorem on the Nevanlinna deficiencies of meromorphic functions of finite order, Ann. of Math. 68 (1958), 203-209.

21. - Proof of a conjecture of G. Polya concerning gap series, Illinois J. Math. 7 (1963), 661-667.

22. W. H. J. Fuchs and W. K. Hayman, An entire function with assigned deficiencies, Studies in Mathematical Analysis and Related Topics, Stanford Univ. Press, Stanford, Calif., 1962, pp. 117-125.

23. A. A. Goldberg, On the inverse problem of the theory of distribution of values of meromorphic functions, Ukrain. Math. J. 6 (1954), 385-397. (Ukrainian)

24. - On the defects of meromorphic functions, Dokl. Akad. Nauk SSSR 98 (1954), 893-895. (Russian)

25. - On a problem in the theory of the distribution of values of meromorphic functions, Dopovidi Akad. Nauk Ukrain. RSR (1954), No. 1. (Ukrainian)

26. - Some asymptotic properties of meromorphic functions, Ucen. Zap. Lvov. Univ. 38 (1956), 54-73. (Russian)

27. - Example of an entire function of finite order with a non-asymptotic deficient value, Učen. Zap. Užgorod. Univ. 18 (1957), 191-194. (Russian)

28. — On meromorphic functions with separated zeros and poles, Izv. Vysš. Ucebn. Zaved. Matematika (1960) No. 4 (17), 67-72. (Russian)

29. - Distribution of values of meromorphic functions with separated zeros and poles, Dokl. Akad. Nauk SSSR 137 (1961), 1030-1033=Soviet Math. Dokl. 2 (1961), 389-392.

30. —, Remark on the theorem of Tumura-Clunie, Dokl. Užgorod Univ., Phys.-Mat. series 1961 (No. 4), 109-110. (Russian)

31. —, On a theorem of M. G. Krein, Dokl. Užgorod Univ., Phys.-Mat. series (1961), 106-108. (Russian)

32. - On the growth of entire functions along a ray, Dokl. Akad. Nauk SSSR 152 (1963), 1049-1050=Soviet Math. Dokl. 4 (1963), 1491-1493.

33. - Three examples of entire functions, Dopovidi Akad. Nauk Ukrainn. RSR (1963), 443-445. (Ukrainian)

34. - On the lower order of an entire function with a finite deficient value, Sibirsk. Mat. Ž. 5 (1964), 54-76. (Russian)

35. - On the size of the lower order of an entire function with a deficient value, Dokl.Akad. NaukSSSR 159 (1964), 968-970=Soviet Math. Dokl. 5 (1964), 1631-1633.

36. A. A. Goldberg and I. V. Ostrovskii, Nerw investigations on the growth and the distribution of values of entire and meromorphic functions of zero, Uspehi Mat. Nauk 16 (1961), 51-62. (Russian)

37. - Some theorems on the growth of meromorphic functions, Ucen. Zap. Kharkov Univ., Series 5 (1961), 3-37. (Russian)

38. A. A. Goldberg and V. G. Tairova, On entire functions with two finite totally ramified values, Zap. Mech. Mat., Faculty of Kharkov University 29, Ser. 4 (1963), 67-78.

39. W. K. Hayman, An integral function with a defective value that is neither asymptotic nor invariant under change of origin, J. London Math. Soc. 28 (1953), 369376.

40. - On the characteristic of functions meromorphic in the unit disk and of their integrals, Acta Math. 112 (1964), 181-214.

41. - On the characteristic of functions meromorphic in the plane and of their integrals, Proc. London Math. Soc. (3) 14a (1965), 93-128. 
42. S. Hellerstein, On a class of meromorphic functions with deficient zeros and poles, Pacific J. Math. 13 (1963), 115-124.

43. B. Kjellberg, On the minimum modulus of entire functions of lower order less than one, Math. Scand. 8 (1960), 189-197. $5-11$.

44. - On the minimum modulus of entire functions, Math. Scand. 12 (1963),

45. T. Kövari, $A$ gap theorem for entire functions of infinite order, Michigan Math. J. 12 (1965), 133-140.

46. B. Ja. Levin and I. V. Ostrovskii, On the dependence of the rate of growth of an entire function on the distribution of the zeros of its derivative (on a question of G. Polya and A. Wiman), Sibirsk. Mat. Ž. 1 (1960), 427-455. (Russian)

47. I. V. Ostrovskii, On meromorphic functions assuming some values at points close to a finite set of rays, Dokl. Akad. Nauk SSSR 120 (1958), 970-972. (Russian)

48. - On the distribution of zeros of the derivative of an entire function all of whose zeros are close to the real axis, Dokl. Akad. Nauk SSSR 130 (1960), 973-976= Soviet Math. Dokl. 1 (1960), 114-117.

49. - On the relation between the growth of a meromorphic function and the angular distribution of its values, Dokl. Akad. Nauk SSSR 132 (1960), 48-52=Soviet Math. Dokl. 1 (1960), 485-488.

50. - Bounds on the deficiencies of meromorphic functions two of whose values are only taken on in an angle, Izv. Vyš̌. Učebn. Zaved. Matematika (1960), 138-148. (Russian)

51. - On the relation between the growth of a meromorphic function and the angular distribution of its values, Izv. Akad. Nauk SSSR 25 (1961), 277-328. (Russian)

52. - On the deficiencies of meromorphic functions of lower order less than one, Dokl. Akad. Nauk SSSR 150 (1963), 32-35=Soviet Math. Dokl. 4 (1963), 587-591.

53. - On a problem of the distribution of values, Dokl. Akad. Nauk SSSR 151 (1963), 34-37.=Soviet Math. Dokl. 4 (1963), 909-913.

54. R. E. A. C. Paley, A note on integral functions, Proc. Cambridge Philos. Soc. 28 (1932), 262-265.

55. V. P. Petrenko, Some estimates of the logarithmic derivative of a meromorphic function, Izv. Akad. Nauk Armjan. SSR 17 (1964), 24-37. (Russian)

56. - On the size of the deficiencies of meromorphic functions, Dokl. Akad. Nauk SSSR 158 (1964), 1030-1033=Soviet Math. Dokl. 5 (1964), 1357-1361.

57. - The growth of a meromorphic function on a ray, Dokl. Akad. Nauk SSSR 155 (1964), 281-284 =Soviet Math. Dokl. 5 (1964), 4.05-408.

58. A. Pfluger, Zur Defektrelation ganzer Funktionen endlicher Ordnung, Comment. Math. Helv. 19 (1946), 91-104.

59. A. Pfluger and G. Pólya, On the power series of an integral function having an exceptional value, Proc. Cambridge Philos. Soc. 31 (1935), 153-155.

60. L. Sario, Value distribution under analytic mappings of arbitrary Riemann surfaces, Acta Math. 109 (1963), 1-10.

61. - General value distribution theory, Nagoya Math. J. 23 (1963), 213-229.

62. Mme. L. Schwartz, Exemple d'une fonction méromorphe ayant des valeurs déficientes non asymptotiques, C. R. Acad. Sci. Paris 212 (1941), 382-384.

63. H. Selberg, Über die Wertverteilung der algebroiden Funktionen, Math. Z. 31 (1930), 709-728.

64. - - Algebroide Funktionen und Umkehrfunktionen Abelscher Integrale, Avh. Norske Vid.-Akad. Oslo, Mat./Naturv. Kl. no. 8 (1934). 
65. D. F. Shea, On the Valiron deficiencies of meromorphic functions of finite order, Trans. Amer. Math. Soc. 124 (1966), 201-227.

66. $\mathrm{O}$. Teichmüller, Vermutungen und Sätze über die Wertverteilung bei gebrochenen Funktionen, Deutsche Math. 4 (1939), 163-190.

67. G. Valiron, Sur le minimum du module des fonctions entières d'ordre inférieur d un, Mathematica 11 (1935), 264-269. 